\title{
EXCITON DYNAMICS IN CdTe/CdMnTe MULTIQUANTUM WELL STRUCTURES GROWN BY MOLECULAR BEAM EPITAXY ON GaAs SUBSTRATE
}

M. Godlewski, B. Koziarska, A. Suchocki, G. Karczewski, T. Wojtowicz, J. Kossut

Institute of Physics, Polish Academy of Sciences

Al. Lotników 32/46, 02-668 Warszawa, Poland

J.P. Bergman and B. Monemar

Dept. of Physics and Meas. Techn., Linköping University, 58183 Linköping, Sweden

The results of picosecond photoluminescence kinetics of four different $\mathrm{CdTe} / \mathrm{CdMnTe}$ multiquantum well structures grown by MBE on GaAs substrates are presented. The experimental results show that excitons in CdTe quantum wells are strongly localized by potential fluctuations. Photoluminescence decay times of the localized excitons are considerably shorter (about $120 \mathrm{ps}$ ) than those reported for free or quasi-free excitons. An influence of $\mathrm{Mn}$ in the barriers on exciton properties is demonstrated. For narrow quantum wells as well as for the multiquantum well structure with the highest Mn mole fraction the excitons migrate during their decay to the states with a lower potential energy. Longer decay times are observed for quasi-localized excitons. We show also that for strongly localized excitons the energy transfer between localized and donor bound excitons is less efficient.

PACS numbers: 78.47.+p, 71.35.+z, 68.60.-p, 73.20.Fz

\section{Introduction}

Exciton dynamics in CdTe/CdMnTe multiquantum well (MQW) structures were studied by several groups [1-5]. Contradictory results were reported with photoluminescence (PL) decay time varying between $100[2,4]$ and $300[5] \mathrm{ps.}$ Also very different exciton properties were reported. A strong red shift of excitons during their decay was observed for structures grown on GaAs substrates and was related to exciton localization at interfaces [1]. In contrast with this result, a strong localization of excitons by potential fluctuations in quantum wells (QWs) was proposed by Yakovlev et al. [2] and Pohlmann et al. [4] for structures grown 
on CdTe (100) substrates. On the other hand, O'Neill et al. observed free excitons (FEs) with $320 \mathrm{ps}(7 \mathrm{~nm} \mathrm{QW})$ decay times for MBE structures grown on InSb and suggested that shorter decay times reported by other groups are likely due to nonradiative recombination [5]. In this paper we present results of detailed study of exciton properties in MBE structures grown on GaAs substrate with [100] growth direction.

\section{Experimental}

Four MBE structures studied were grown on (100) GaAs substrate with Mn fraction in the barriers 10\% (sample \#09203), 30\% (\#09213), 51\% (\#09223) and $68 \%$ (\#09233), respectively. GaAs substrate was covered with $0.2 \mu \mathrm{m} \mathrm{ZnTe}$, $0.8 \mu \mathrm{m} \mathrm{CdTe}$ and $2 \mu \mathrm{m} \mathrm{Cd}{ }_{1-x} \mathrm{Mn}_{x} \mathrm{Te}$ buffer layers followed by four (three (4, 6 and $10 \mathrm{~nm}$ ) in the case of the structure with $10 \%$ of $\mathrm{Mn}$ ) QWs of 2, 4,6 and $10 \mathrm{~nm}$ thickness separated by $50 \mathrm{~nm} \mathrm{Cd}{ }_{1-x} \mathrm{Mn}_{x} \mathrm{Te}$ barrier and $100 \mathrm{~nm}$ CdMnTe cap layer with the same value of $x$ as in the barriers. All structures studied had [100] growth direction.

PL and PL excitation (PLE) spectra were measured at $2 \mathrm{~K}$ on the conventional setups. Exciton dynamics was studied with a Hamamatsu synchroscan camera with a temporal resolution of about $20 \mathrm{ps}$. Pulsed excitation was provided by a dye laser synchronously pumped with a mode-locked argon laser. Pulse length was about 5 ps.

\section{Results}

PL spectrum of MQW structures studied consisted of a set of PL lines due to radiative recombination in each of the QWs. A doublet structure of PL lines was observed and was related to simultaneous observation of localized (LE) and neutral donor bound (DBE) excitons. A weak acceptor bound exciton PL was observed in some cases. Detailed PL kinetics investigations will be presented for the wide QWs $(4,6$ and $10 \mathrm{~nm})$. For $2 \mathrm{~nm}$ QW a polaronic effect strongly contributed to the exciton dynamics. Magnetic polaron formation was discussed recently by several authors $[6,7]$ and will not be discussed here.

PL decay times were in the range of $100-140$ ps for the LEs and slightly longer for DBEs (140-180 ps). With an increase in temperature PL decay time rapidly increases and reaches about $350 \mathrm{ps}$ for $60 \mathrm{~K}$ temperature. In Fig. 1 we show 2D contour plot of the PL intensity versus emission energy and decay time for the $6 \mathrm{~nm} \mathrm{QW}$ of the CdTe/CdMnTe structure with $10 \%$ of Mn in the barriers. For the $6 \mathrm{~nm}$ QW PL decays without any shift of its spectral position. However, a small spectral shift was observed for the $4 \mathrm{~nm}$ QW in the same structure. These exciton properties can be compared with the one shown in Fig. 2 for the $10 \mathrm{~nm}$ thick QW in the structure grown with $68 \%$ of $\mathrm{Mn}$ in the barrier.

PLE spectra were measured for all structures studied. Stokes shifts between $\mathrm{PL}$ and PLE peaks depended on the Mn mole fraction in the barriers and varied between $2 \mathrm{meV}(10 \% \mathrm{Mn})$ and $3-5 \mathrm{meV}(51 \% \mathrm{Mn})$. The latter was similar to that reported by Zhang et al. [1]. The PLE peaks had quite different widths. For narrow QWs and for MQW structures with large Mn mole fraction in the barriers the PLE peaks were relatively broad. 


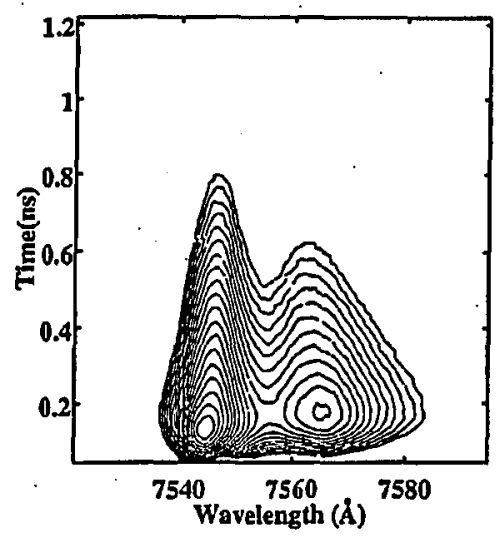

Fig. 1. 2D contour plot of the PL intensity versus emission energy and decay time for $6 \mathrm{~nm} \mathrm{QW}$ in CdTe/CdMnTe MBE structure with $10 \%$ of Mn in the barriers.

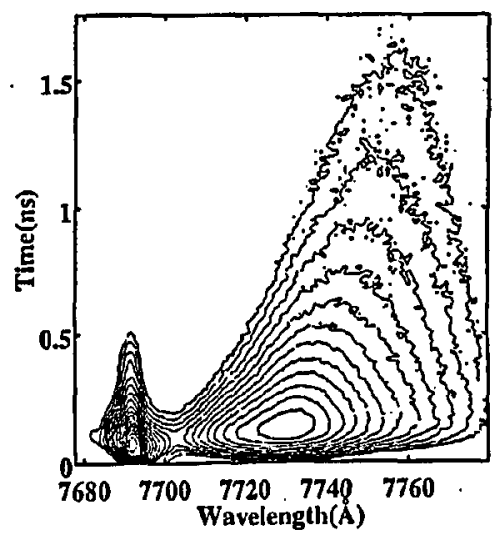

Fig. 2. 2D contour plot of the PL intensity versus emission energy and decay time. for $10 \mathrm{~nm}$ thick $\mathrm{QW}$ in CdTe/CdMnTe MBE structure grown with $68 \%$ of $\mathrm{Mn}$ in the barriers.

\section{Discussion}

The transition probability of excitons in QWs is proportional to the area of coherent excitation. This is why short decay times are expected for FEs (about $10 \mathrm{ps}$ ), since FEs in perfect structures are coherent excitations of the whole crystal [8]. In a reality PL decay times of FEs observed in the experiment are considerably longer since FEs have a finite coherence length due to dephasing scattering processes. They reach $300 \mathrm{ps}$ for FEs in $\mathrm{CdTe} / \mathrm{CdMnTe} \mathrm{MBE}$ structures [5]. On the other hand, the coherent area of LEs is determined by a spatial extent of localized states. Therefore, their radiative decay time should also be longer than the theoretical radiative decay time of FEs. The differences in the PL decay times of LEs and FEs result thus from differences in the coherence lengths of both types of excitons. Due to the finite scattering times PL decay times can be quite different 
from the theoretical one and can vary from sample to sample or even for QWs of different width in the same MBE structure.

Such property of excitons in QWs is confirmed by the present study. The PLE experiment (Stokes shift) and the observed decay of PL without spectral shift prove a localized character of excitons in wide $(6$ and $10 \mathrm{~nm}) \mathrm{QWs}$ and for structures with a small Mn fraction in the barrier. In the high resolution PL study and in the PLE experiment we have observed relatively narrow emission lines for these QWs. This indicates relatively small density of localized states in the QW structures. PL kinetics experiment shows that free excitons are first formed under photo-excitation. They convert to the LEs in times between 20 and 50 ps. With an increase in temperature LEs can delocalize and the PL decay times increase rapidly to the values reported for FEs. We report also that for LEs energy transfer to DBEs is less efficient than for FEs.

For relatively narrow $(4 \mathrm{~nm}) \mathrm{QW}$ the strain built into the structure results in a slightly increased density of localized states. LEs in such QWs even after their initial localization can have some excess potential energy and can migrate between various localized states. The process is assisted by either absorption or emission of an acoustic phonon. Phonon-assisted exciton migration was observed previously for narrow GaAs/AIGaAs QWs [9]. PL and PLE spectra are then wider indicating increasing density of the localized states in the narrow QWs.

Similar results were obtained for the MQW structures grown with the $10 \%$, $30 \%$ and $51 \%$ of $\mathrm{Mn}$ in the barriers. A distinctly different exciton decay patterns were observed for the structure with the $68 \%$ of $\mathrm{Mn}$ in the barriers. Excitons in this case are not strongly localized and they are quasi-mobile. Figure 2 shows a strong spectral shift of the PL of $10 \mathrm{~nm}$ QW within its decay time. PL and PLE spectra are both relatively wide (4-5 meV) proving an increased density of localized states.

\section{Conclusions}

The present study proves a localized nature of excitons in wide QWs of $\mathrm{CdTe} / \mathrm{CdMnTe}$. For narrow QWs and for structures with large Mn fraction in the barriers excitons become quasi-mobile. A phonon assisted exciton migration between localized states is observed. We conclude also that scatter of reported PL decay times reflects different coherent area of excitons in QW studied rather than influence of nonradiative recombination processes.

This work was partly supported by the grant PBZ-Z011/P4/93/01 of the State Committee for Scientific Research (Republic of Poland).

\section{References}

[1] X.-C. Zhang, S.-K. Chang, A.V. Nurmikko, L.A. Kolodziejski, R.L. Gunshor, S. Datta, Appl. Phys. Lett. 47, 59 (1985).

[2] D.R. Yakovlev, W. Ossau, G. Landwehr, R.N. Bicknell-Tassius, A. Waag, I.N. Uraltsev, Solid State Commun. 76, 325 (1990).

[3] M.J. McNamee, W. Hayes, D.J. Mowbray, J.F. Ryan, D.E. Ashenford, B. Lunn, J. Lumin. 48/49, 755 (1991). 
[4] A. Polhmann, R. Hellmann, E.O. Göbel, D.R. Yakovlev, W. Ossau, A. Waag, R.N. Bicknell-Tassius, G. Landwehr, Appl. Phys. Lett. 61, 2929 (1992).

[5] M. O'Neill, M. Oestreich, W.W. Rühle, D.E. Ashenford, Phys. Rev. B 48, 8980 (1993).

[6] E.O. Göbel, R. Hellmann, G. Mackh, D.R. Yakovlev, W. Ossau, A. Waag, G. Landwehr, Workshop on Diluted Magnetic Semicond., Linz 1994, Mater Sci. Forum, in press.

[7] T. Dietl, P. Peyla, W. Grieshaber, Y. Merle d'Aubigné, Phys. Rev. Lett. 74, 474 (1995).

[8] E. Hanamura, Phys. Rev. B 38, 1228 (1988).

[9] M. Zachau, J.A. Kash, W.T. Masselink, Phys. Rev. B 44, 8403 (1991). 